\title{
Cell engineering method using fluorogenic oligonucleotide signaling probes and flow cytometry
}

\author{
Kambiz Shekdar (D) · Jessica Langer • Srinivasan Venkatachalan • \\ Lori Schmid • Jonathan Anobile • Purvi Shah • Amy Lancaster • \\ Olga Babich $\cdot$ Olga Dedova $\cdot$ Dennis Sawchuk
}

Received: 1 October 2020/ Accepted: 5 February 2021/Published online: 8 March 2021

(C) The Author(s), under exclusive licence to Springer Nature B.V. part of Springer Nature 2021

\begin{abstract}
Objective Chromovert ${ }^{\circledR}$ Technology is presented as a new cell engineering technology to detect and purify living cells based on gene expression.

Methods The technology utilizes fluorogenic oligonucleotide signaling probes and flow cytometry to detect and isolate individual living cells expressing one or more transfected or endogenously-expressed genes.

Results Results for production of cell lines expressing a diversity of ion channel and membrane proteins are presented, including heteromultimeric epithelial sodium channel $(\alpha \beta \gamma-\mathrm{ENaC})$, sodium voltage-gated ion channel 1.7 (NaV1.7- $\alpha \beta 1 \beta 2)$, four unique $\gamma$ aminobutyric acid $\mathrm{A}\left(\mathrm{GABA}_{\mathrm{A}}\right)$ receptor ion channel subunit combinations $\alpha 1 \beta 3 \gamma 2 \mathrm{~s}, \alpha 2 \beta 3 \gamma 2 \mathrm{~s}, \alpha 3 \beta 3 \gamma 2 \mathrm{~s}$ and $\alpha 5 \beta 3 \gamma 2 \mathrm{~s}$, cystic fibrosis conductance regulator (CFTR), CFTR- $\Delta 508$ and two G-protein coupled receptors (GPCRs) without reliance on leader
\end{abstract}

K. Shekdar $(\bowtie) \cdot$ J. Langer · S. Venkatachalan .

L. Schmid · J. Anobile . P. Shah .

A. Lancaster - O. Babich · O. Dedova - D. Sawchuk

Chromocell Corporation, North Brunswick,

NJ, USA

e-mail: kambiz.shekdar@secondcellbio.com

K. Shekdar

Secondcell Bio, LLC, New York, NY, USA

D. Sawchuk

D3 Insight Consulting, LLC, Fanwood,

NJ, USA sequences and/or chaperones. In addition, three novel plasmid-encoded sequences used to introduce $3^{\prime}$ untranslated RNA sequence tags in mRNA expression products and differentially-detectable fluorogenic probes directed to each are described. The tags and corresponding fluorogenic signaling probes streamline the process by enabling the multiplexed detection and isolation of cells expressing one or more genes without the need for gene-specific probes.

Conclusions Chromovert technology is provided as a research tool for use to enrich and isolate cells engineered to express one or more desired genes.

Keywords Cell engineering - Genetic engineering · Flow cytometry $\cdot$ Fluorescence-activated cell sorting (FACS) · Cell line · GPCR · Ion channel

\section{Introduction}

Mammalian cell lines, especially those produced using immortalized lines like human embryonic kidney 293 (HEK 293) and Chinese hamster ovary (CHO) cells, are widely used in biological research, including in drug discovery and to produce biologic drugs. In general, cell line production begins with the introduction of one or more DNA sequences of interest into a cell culture resulting in a diversity of genetically distinguished cells based on the site and copy number of integration events. The goal is to produce, in a 
reasonable time frame, clonal cell lines that meet desired criteria for an application of interest.

Despite multiple advances in cell engineering including selection of HEK 293 and CHO host cell subtypes with improved functionality, e.g., HEK 293 T cells (Pear et al. 1993) and serum-free, suspension adapted DG44 CHO cells (Urlaub et al. 1983), cotransfection and/or tagging of DNA sequences of interest with markers including autofluorescent proteins such as green fluorescent protein, GFP (Chalfie et al. 1994), N-terminal tagging of protein expression products with leader sequences (e.g., using rhodopsin leader sequences) to enhance folding, assembly and/or membrane integration of protein expression products (Krautwurst et al. 1998), the addition of chaperones (Mohan et al. 2007) and strategies to amplify the copy number of genetic recombination events (Cockett et al. 1990), the engineering of cells treated to comprise one or more added genes of interest remains challenging even given precision genetic engineering tools such as clustered regularly interspaced short palindromic repeats, or CRISPR (Nowogrodzki 2019). For instance, the use of cell lines to produce recombinant adeno-associated virus (rAAV) as required for numerous approved gene therapies has been limited due to the unpredictable and overly timeconsuming nature of the process even though cell lines are highly sought-after to replace current rate-limiting rAAV production methods that involve transient transfection of multiple plasmids into HEK 293T cells (Wang et al. 2019).

Here, we present Chromovert technology as a research tool for production of mammalian cell lines comprising one or more sequences of interest. The method is based upon the broadly-applicable principles of fluorescence-resonance energy transfer (FRET) and nucleic acid hybridization using fluorogenic oligonucleotide signaling probes originally reported for in tubo qRT-PCR applications (Marras et al. 1999) transfected into living cells. The termini of the signaling probes are covalently linked to a fluorophore or quencher paired to absorb its emission. The termini are designed to form a 4-7 base-pair stem juxtaposing the fluorophore and quencher pair. In the presence of target sequence, hybridization of the sequence-specific probe results in a fluorogenic conformational change. Flow cytometry is then used to detect and isolate positive cells that fluoresce above background. Thousands of individual clones can then
Fig. 1 Detection of gene expression in individual living cells using Chromovert technology. a Three artificial RNA sequences, termed Chromo-Tags or C-Tags A, B and C, used to tag mRNAs of interest for detection using signaling probes are shown. Color-coding is used to indicate sequence variations between Chromo-Tag A and either Chromo-Tag B or C. The Chromo-Tags vary from each other by at least $50 \%$ within the 24-nucleotide region indicated. b Predicted RNA folding for Chromo-Tags A, B and C using the mFold algorithm is shown. c Detection of a target RNA by a signaling probe in transfected cells vs. control-transfected cells using fluorescence microscopy is shown on the left and right panels, respectively. Scale bar $=100 \mu \mathrm{m}$. d Cell cytometric analysis for the detection of Chromo-Tags A and B using differentially-labeled signaling probes to Chromo-Tags A, B and C in transfected cells (blue traces) versus control-transfected cells (red traces) is shown. Fluorescence above background corresponding to signaling probes directed to Chromo-Tags A and B is shown in the left and center panels, respectively. Background fluorescence corresponding to control signaling probe directed to Chromo-Tag $\mathrm{C}$ is shown in the right panel. $X$-axis: fluorescence signal corresponding to the indicated probe, $Y$-axis: normalized cell count. e 2D dot plots for the simultaneous detection of Chromo-Tags A and B in transfected cells (left panel) versus control-transfected cells (right panel) are shown. Percentages of cells falling in each quadrant are indicated. $X$-axis and $Y$-axis: fluorescence signal corresponding to the indicated probe. $\mathbf{f}$ Far left panel shows single-point HTS screening data for cell lines expressing the human bitter taste receptor GPCR T2R16 ("Native") or the T2R16 comprising amino acids 1-39 of rhodopsin as an N-terminal tag ("Rhodo") in response to milkweed extract using a fluorescent calcium flux cell-based assay, as indicated. Center left panel shows a dose-response cure for Helional in the native human odorant receptor OR3A1 cell line ("hOR3A1") using a calcium flux cell-based assay, as indicated. Center right panel shows a dose-response curve for the inhibition of current in the NaV1.7- $\alpha \beta 1 \beta 2$ stable cell line by a small molecule compound CC8464 ( $\mathrm{n}=7)$. X-axis: CC8464 concentration, Y-axis: fraction of normalized current blocked using a wholecell patch-clamp assay. Far right panel shows the response of the GABA $_{\mathrm{A}} \alpha 1 \beta 3 \gamma 2 \mathrm{~s}, \alpha 2 \beta 3 \gamma 2 \mathrm{~s}, \alpha 3 \beta 3 \gamma 2 \mathrm{~s}$ and $\alpha 5 \beta 3 \gamma 2 \mathrm{~s}$ ion channel cell lines to GABA using a fluorescent membrane potential cellbased assay, as indicated

be isolated and expanded using automated cell culture methods. Functional testing over time in the absence of selective pressure is used to select final clones.

\section{Results}

Data are presented for the production of 11 unique cell lines expressing ion channel or GPCR proteins. The cell lines were produced to express a total of 26 transfected cDNAs. All cell lines were prepared using the same standardized protocol without any gene- 
a A GCAGGUGGACAGGAAGGUUCUAAUGUUCUUAAGGCACAGGAACUGGGACAUCUGGGCCCGGAAAGCCUUUUUCUCUGUGAUCCGGUACAGUCCUUCUGC

B GCAGGUGGACAGCUUGGUUCUAAUGAAGUUACCCUGUCGUUCUGGACAUCUGGGCCCGGAAAGCGUUAACUGAUGGAU -- GGAACAGUCCUUCUGC

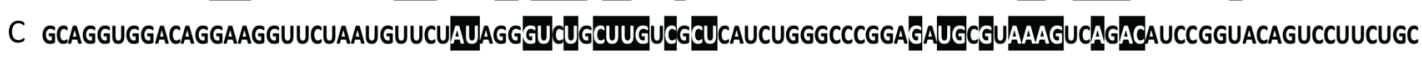

b
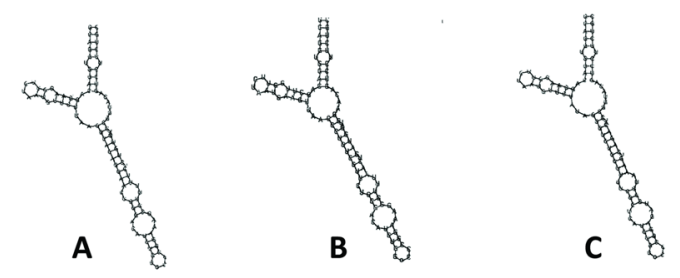

C
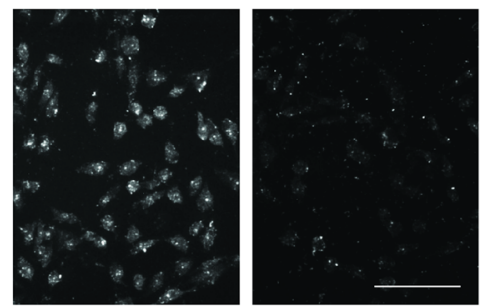

d

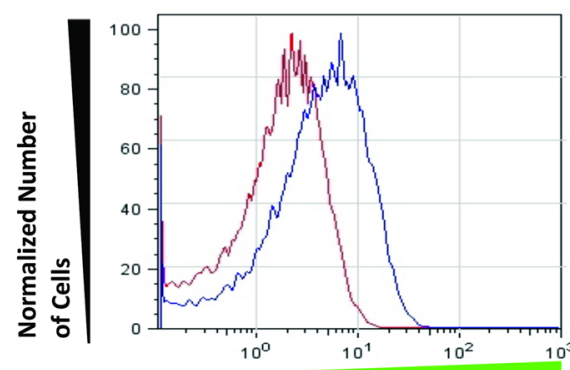

Probe 1

e

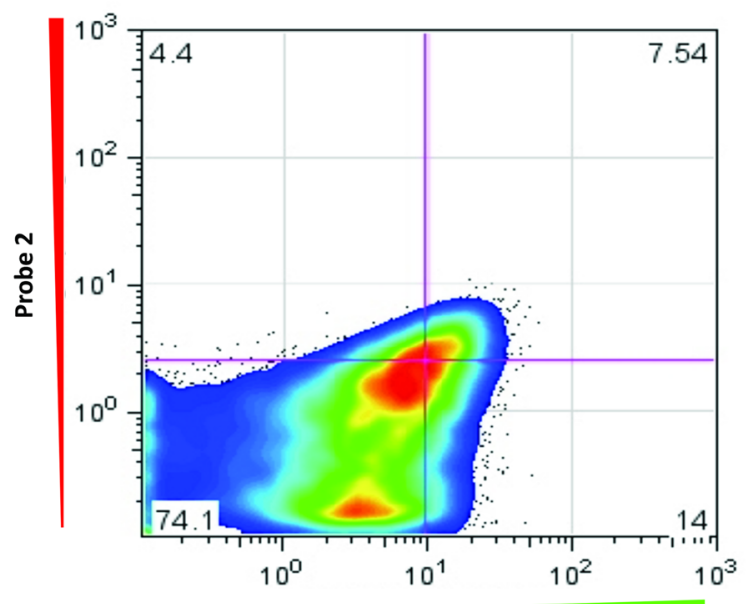

Probe 1
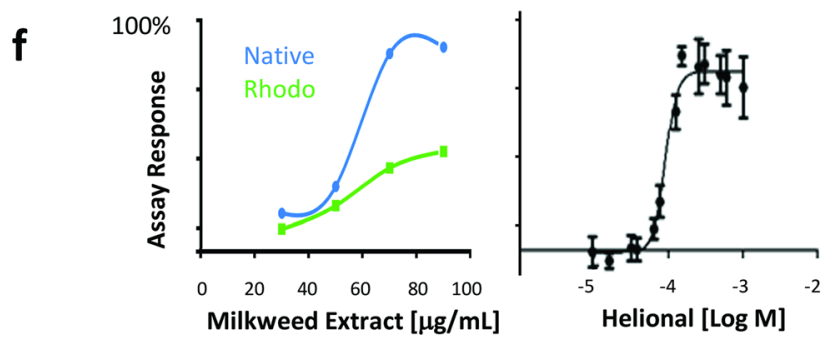

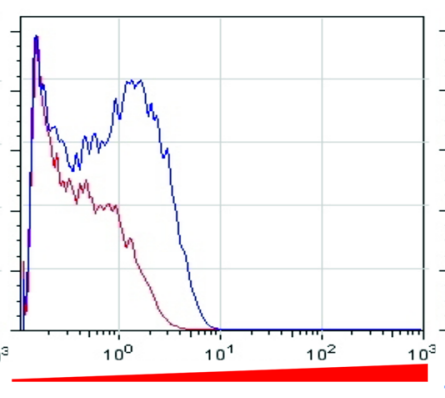

Probe 2

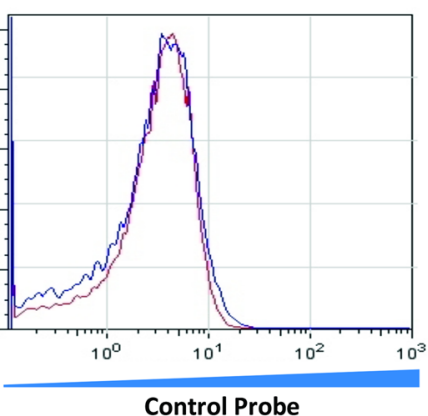

Control Probe

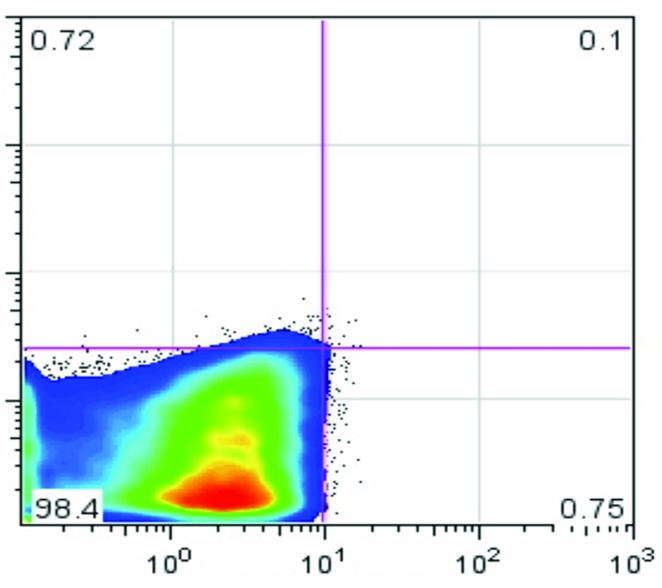

Probe 1

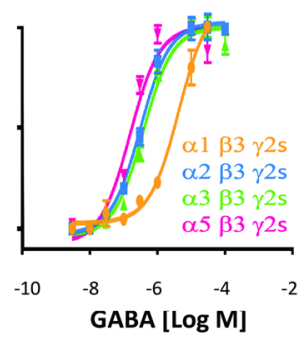


specific signaling probes. Generic signaling probes directed to three plasmid-encoded sequence tags, termed Chromo-Tags ${ }^{\mathrm{TM}}$, were used to detect and isolate cells expressing any cDNAs required to produce all the cell line described in this report. Three Chromo-Tags that vary by at least $50 \%$ in pairwise comparisons over the 24-nucleotide region indicated and that share similar predicted mRNA folding based on the mFold algorithm (Zuker 2003) were designed, as shown in Fig. 1a and b, respectively. Representative data for the multiplexed detection of the three Chromo-Tag sequences expressed in cells co-transfected with expression plasmids each comprising an expression cassette encoding one of the Chromo-Tags using differentially-labeled signaling probes directed to the 24-nucleotide region of the Chromo-Tags are shown in Fig. 1c-e.

Chromovert technology was used to produce cell lines expressing GPCRs. Figure 1f, far left and center left panels show calcium flux cell-based assay results for three GPCR cell lines produced using the Chromovert method. Functional cell-based responses for the cell line comprising the native human bitter taste receptor T2R16 and a second cell line comprising a modified version of the same GPCR in response to milkweed extract are shown in Fig. 1f, far left panel. The modified T2R26 comprises an N-terminal amino acid extension corresponding to amino acids $1-39$ of rhodopsin previously reported to be required for the functional expression of human bitter GPCRs (Chandrashekar et al. 2000). Both cell lines were cotransfected to express the promiscuous murine G-alpha protein, $\mathrm{G} \alpha_{15}$ (Offermanns and simon 1995). Figure 1f, center left panel shows the response of the Chromovert technology-enabled cell line expressing the native human odorant GPCR hOR31A and $\mathrm{G} \alpha_{15}$ to $\alpha$-methyl-3,4-methylendioxy-hydrocinnamal-dehyde, or Helional. The hOR3A1 cell line was also produced without reliance on any chaperones or N-terminal amino acid leader sequences.

Chromovert technology was used to produce cell lines expressing ion channels. Figure 1f, center right and far right panels show cell-based assay results for five heteromultimeric ion channel cell lines produced using Chromovert technology and Chromo-Tags. The functional cell-based response of the NaV1.7 cell line to a NaV1.7 inhibitor, Chromocell compound CC8464 (Babich et al. 2016), is shown in Fig. 1f, center right panel. A combinatorial panel of cell lines and cell- based assays for four $\mathrm{GABA}_{\mathrm{A}}$ receptor ion channel subunit combinations $\alpha 1 \beta 3 \gamma 2 \mathrm{~s}, \alpha 2 \beta 3 \gamma 2 \mathrm{~s}, \alpha 3 \beta 3 \gamma 2 \mathrm{~s}$ or $\alpha 5 \beta 3 \gamma 2$ s was also generated, as shown in Fig. 1f, far right panel. High-throughput screening (HTS) of a library of pharmaceutically active compounds (LOPAC, Sigma 1280) was performed using the panel. We identified 34 LOPAC compounds that activated at least one of the cell lines at least as much as GABA itself, including all known $\mathrm{GABA}_{\mathrm{A}}$ agonists included in LOPAC: GABA, Propofol, Isoguvacine hydrochloride, Muscimol hydrobromide, Piperidine4-sulphonic acid, 3-alpha, 21-dihydroxy-5-alphapregnan-20-one, 5-alpha-pregnan-3alpha-ol-11,20dione, 5-alpha-pegnan-3alpha-ol-20-one and Tracazolate (Johnston 2005). In addition, at least four compounds not previously described as $\mathrm{GABA}_{\mathrm{A}}$ agonists but known to have other activities associated with $\mathrm{GABA}_{\mathrm{A}}$ ion channels were identified, including: etazolate, androsterone, chlormezanone and ivermectin. The screen further identified compounds which, until now, were not reported to interact with $\mathrm{GABA}_{\mathrm{A}}$, including: dipyridamole, niclosamide, tyrphostin A9 and I-OMe-Tyrphostin AG 538.

Chromovert technology was also used to produce CFTR and CFTR- $\Delta 508$ cell lines and cell-based assays. Figure $2 \mathrm{a}$, left panel shows a dose-response curve for the CFTR cell line to Forskolin. Figure 2a, right panel shows that the Chromovert technologyenabled CFTR- $\Delta 508$ cell line enables a functional cell-based assay for CFTR- $\Delta 508$ in the absence of any added trafficking correctors. Figure $2 b$ shows the functional cell-based responses of multiple Chromovert technology-enabled CFTR cell lines compared to transiently-transfected cells.

Finally, Chromovert technology was used to create a cell line for ENaC. ENaC causes cell swelling and is cytotoxic when expressed in transfected cell cultures, as shown in Fig. 3a. Chromovert technology enabled an $\alpha \beta \gamma$-ENaC cell line as shown in Fig. 3b using a cell-based assay selective for $\alpha \beta \gamma-\mathrm{ENaC}$ activity. Limited proteolysis of the cell line was used to achieve functionally distinct proteolyzed variants, as shown in Fig. 3c. HTS of a compound library against nonproteolyzed versus proteolyzed $\mathrm{ENaC}$ variants resulted in largely non-overlapping hits, as shown in Fig. 3d. One potentiator identified during HTS of proteolyzed $\mathrm{ENaC}$ and confirmed to enhance the salty taste of sodium chloride using human sensory studies is shown in Fig. 3e. 

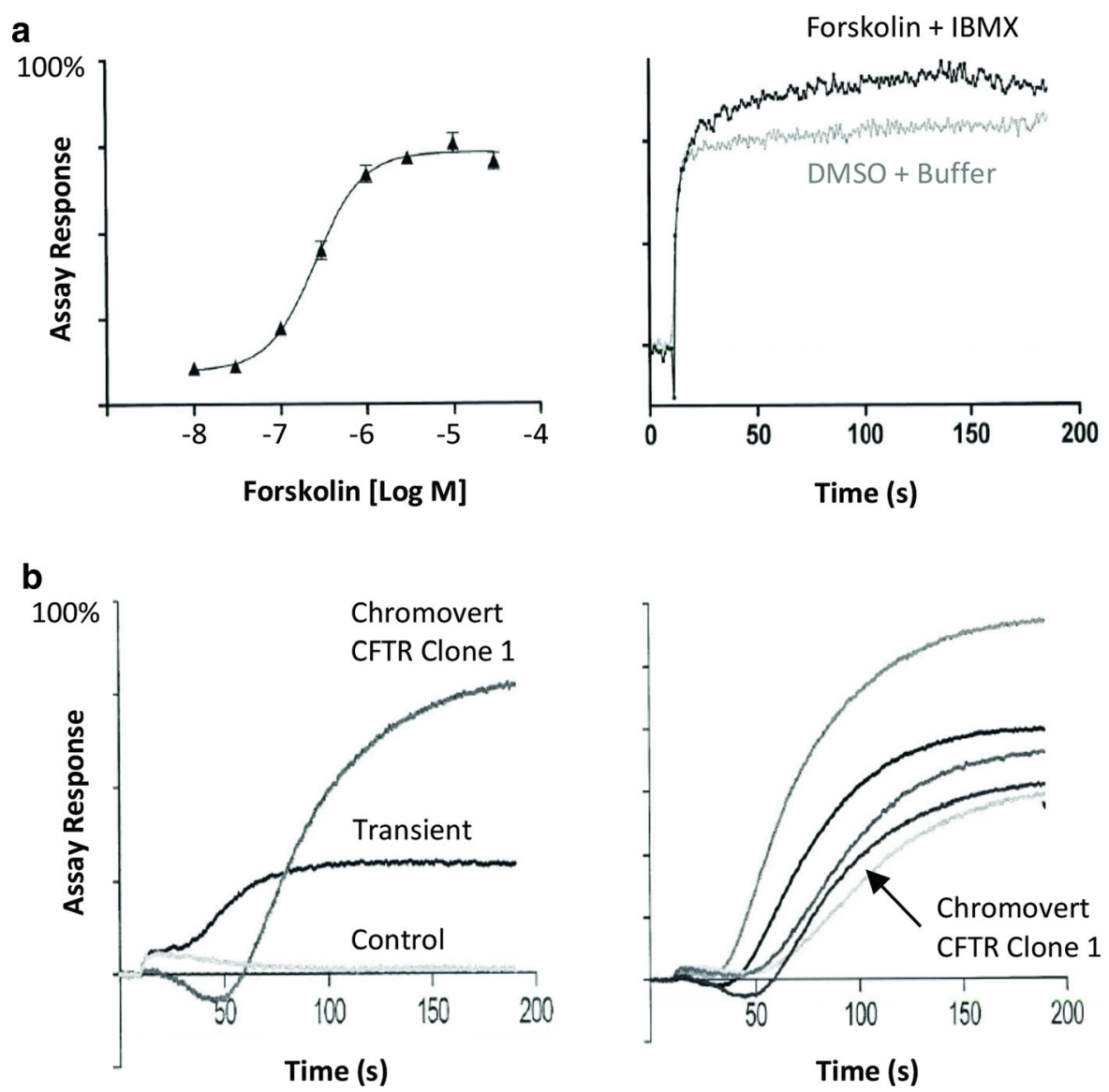

Fig. 2 Comparison of Chromovert technology-enabled CFTR cell line to traditional results using a fluorescent membrane potential assay. a Left panel shows the dose response curve of the CFTR cell line to forskolin, resulting in an $\mathrm{EC}_{50}$ value of $256 \mathrm{nM}$. Right panel shows the response of the CFTR- $\Delta 508$ cell line to $30 \mu \mathrm{M}$ Forskolin $+100 \mu \mathrm{M}$ IBMX (3-isobutyl-1methyl xanthine) versus DMSO + Buffer in the absence of

\section{Discussion}

Chromovert technology utilizes fluorogenic oligonucleotide signaling probes to detect and isolate individual cells expressing one or more genes of interest, see technology flowchart in Fig. 4. The technology was used to create cell lines for a diversity of proteins. All the cell lines in this report were created using only signaling probes directed to plasmid-encoded RNA tags, termed Chromo-Tags. Chromo-Tags are 3' untranslated RNA sequence tags subcloned for expression downstream of the stop codon of cDNAs of interest. Unlike epitope tags, Chromo-Tags are not translated into protein such that the amino acid sequences of expression products are not altered.

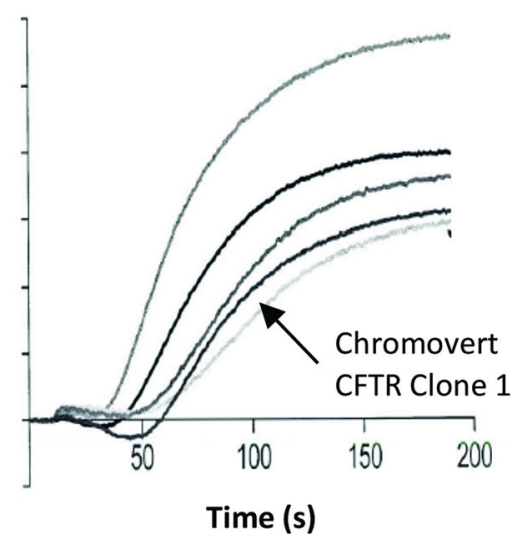

any trafficking correctors. b Left panel shows the response of the one Chromovert CFTR cell line (Clone 1), cells transientlytransfected with CFTR and control-transfected cells to an $\mathrm{EC}_{50}$ concentration of Forskolin. Right panel shows the response of Clone 1 compared to additional Chromovert CFTR clonal cell line isolates

Chromo-Tags streamline the cell engineering method by obviating the need to design and develop gene-

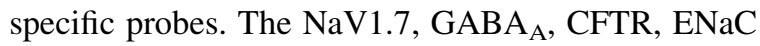
and GPCR example cell lines were all produced using the same Chromo-Tags and protocols. Whereas numerous cell-based approaches have been reported to access each of these proteins, the Chromovert technology-enabled cell lines and cell-based assays contributed to research and development efforts relating to each of these proteins, as described below.

First, whereas GPCRs are commonly reported to require $\mathrm{N}$-terminal amino acid extensions (e.g., leader sequences derived from rhodopsin) and/or chaperones for functional expression in cultured cells (Krautwurst et al. 1998; Mohan et al. 2007), the native and full- 
a
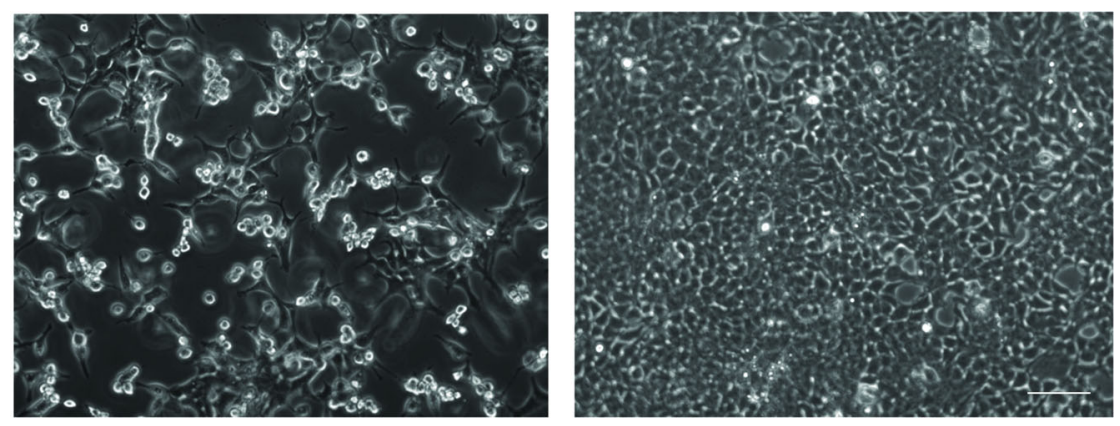

b
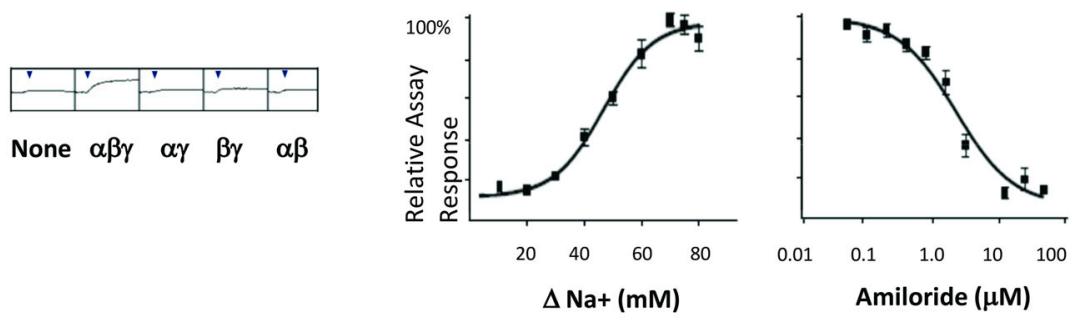

C

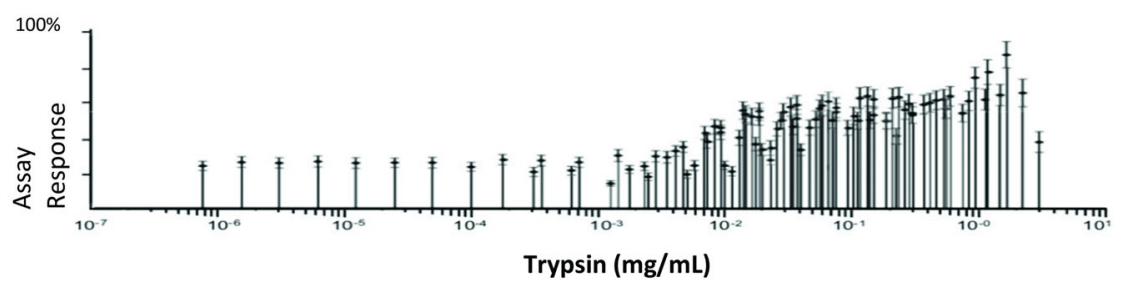

d

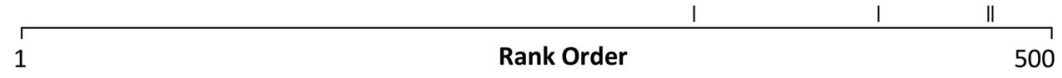

e

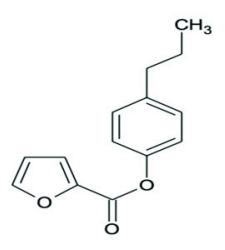

STE 1

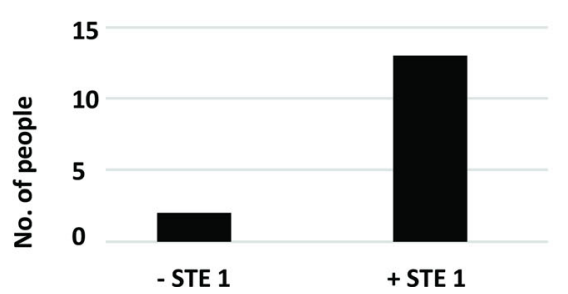

length functional GPCR cell lines in this report were produced using neither. In the case of human odorant receptor hOR3A1, the Chromovert technology-enabled cell line for the native receptor matched literature results (Wetzel et al. 1999). In the case of human bitter taste receptor T2R16, our results indicate that $\mathrm{N}$-terminal rhodopsin leader sequences may in some cases affect the physiological form and function of the native receptors.
Second, NaV1.7 inhibitors are highly sought as non-addictive pain blockers for use to help address the opioid epidemic however stable cell lines comprising multiple accessory subunits have not been reported. The NaV ion channel family is comprised of large pore-forming $\alpha$-subunits and up to four accessory $\beta$ subunits, $\beta 1, \beta 2, \beta 3$ and $\beta 4$, which modulate their activity (O'Malley and Isom 2015). HTS of the Chromovert technology-enabled NaV1.7- $\alpha \beta 1 \beta 2$ cell 
4Fig. 3 Creation and testing of a stable $\alpha \beta \gamma$-ENaC cell line. a Expression of $\alpha \beta \gamma$-ENaC is cytotoxic in transiently-transfected cultured cells (left) versus control-transfected cells (right) as visualized by phase-contrast microscopy. Scale bar $=100$ $\mu \mathrm{m}$. b Left panel shows a functional cell-based assay selective for $\alpha \beta \gamma$-ENaC using cells transiently-transfected cells. $X$-axis: time, Y-axis, assay response. Arrows: addition of solution to increase sodium by $75 \mathrm{mM}$. Right panel shows functional characterization of the Chromovert technology-enabled ENaC cell line using the assay. Responses to increasing changes in sodium concentration and inhibition of the response to a $75 \mathrm{mM}$ increase in sodium concentration by amiloride are shown. c Multiple functionally-distinct proteolyzed variants of $\alpha \beta \gamma$ $\mathrm{ENaC}$ created using limited proteolysis in the context of the cellbased assay are shown $(n=9)$. d The only four compounds among the top 500 hits identified in HTS of a compound library screened using proteolyzed $\mathrm{ENaC}$ that were also identified in HTS of the same library screened using non-proteolyzed ENaC are rank-ordered for their activity on non-proteolyzed ENaC. e The chemical structure and human sensory taste testing results for 4-propylphenyl 2-furoate, a potentiator of proteolyzed $\alpha \beta \gamma$ ENaC identified using HTS termed salt-taste enhancing compound 1 (STE1), are shown. In pairwise comparisons of $45 \mathrm{mM} \mathrm{NaCl} \pm 15 \mu \mathrm{g} \mathrm{STE} 1 / \mathrm{mL}$ using a human sensory taste testing protocol approved by an institutional review board, 13 out of 15 participants selected the sample containing STE1 as more salty

line resulted in the discovery and development of Chromocell compound CC8464 (Babich et al. 2016), a clinical lead compound that has been advanced through human Phase I studies with potential to address the opioid epidemic.

Third, results using the Chromovert technologyenabled panel of four $\mathrm{GABA}_{\mathrm{A}}$ cell lines provide proofof-concept data for the production of more comprehensive combinatorial panels of $\mathrm{GABA}_{\mathrm{A}}$ subunits for use to increase understanding of subunit combinations that underlie in vivo function and drug pharmacology. The $\mathrm{GABA}_{\mathrm{A}}$ ion channel family includes at least 19 different subunits ( 6 alpha, 3 beta, 3 gamma, 1 delta, 1 epsilon, 1 theta, 1 pi, and 3 rho subunits) thought to form pentameric ion channels comprising 2 alpha subunits, 2 beta subunits and a third subunit (Olsen and Sieghart 2008). Drugs, including FDA-approved drugs and failed candidate molecules, may be profiled against such panels to identify in vitro correlates for desired pharmacology and unwanted side-effects.

Fourth, comparisons of Chromovert technologyenabled CFTR and CFTR- $\Delta 508$ cell lines suggest certain advantages compared to traditional cell-based assays. For instance, whereas transient transfection is generally regarded to produce more robust cell-based assay results as compared to cell lines, Chromovert technology enabled cell lines with 2 to 6 fold or more functionality. Previously, no CFTR- $\Delta 508$ cell lines have been reported to result in cell-based assay for the detection of CFTR- $\Delta 508$ in the absence of trafficking correctors used to release the mutant CFTR trapped in intracellular compartments. In contrast, the Chromovert technology-enabled CFTR- $\Delta 508$ cell line resulted in a detectable cell-based assay signal in the absence of any trafficking correctors. This CFTR mutant is the most prevalent in cystic fibrosis (Lemna et al. 1990). The creation of a cell-based assay for CFTR- $\Delta 508$ that does not necessitate the use of trafficking correctors may provide for more physiologically relevant drug discovery.

Finally, $\mathrm{ENaC}$ is a highly-sought heteromultimeric ion channel drug target comprising $\alpha, \beta$ and $\gamma$ subunits expressed on the apical surface of epithelial cells where it conducts sodium and plays a role in fluid balance (Fornius 2013). In the lung, ENaC is a validated drug target in pulmonary edema (PE), chronic obstructive pulmonary disease (COPD) and cystic fibrosis $(\mathrm{CF})$, with potential to treat coronavirus disease 2019, COVID-19, (Matalon et al. 2015; Eisenhut and Shin 2020). ENaC has also been postulated as the putative human salt taste receptor (Roper 2015), where salt taste enhancers could be used to reduce dietary sodium. However, cell-based drug discovery against this validated drug target has been hampered due to its cytotoxicity in cultured cells. Only Chromovert technology enabled the creation of an $\alpha \beta \gamma-\mathrm{ENaC}$ cell line. The creation of the $\mathrm{ENaC}$ cell line in turn enabled limiting proteolysis to achieve functionally distinct proteolyzed variants, resulting in the discovery of an $\mathrm{ENaC}$ potentiator and human sensory confirmation of ENaC's role as a human salt taste receptor.

Many cultured cells exhibit vast cell-to-cell genetic diversity. For example, according to the product specifications at ATCC at https://www.atcc.org/, the HEK 293 cell line is characterized as follows: "This is a hypotriploid human cell line. The modal chromosome number was 64 , occurring in $30 \%$ of cells. The rate of cells with higher ploidies was $4.2 \%$." Compared to other cell line production methods, it is possible that the signaling probes used in Chromovert technology may exploit the heterogeneity of transfected cell cultures by serving as biomarkers to detect 

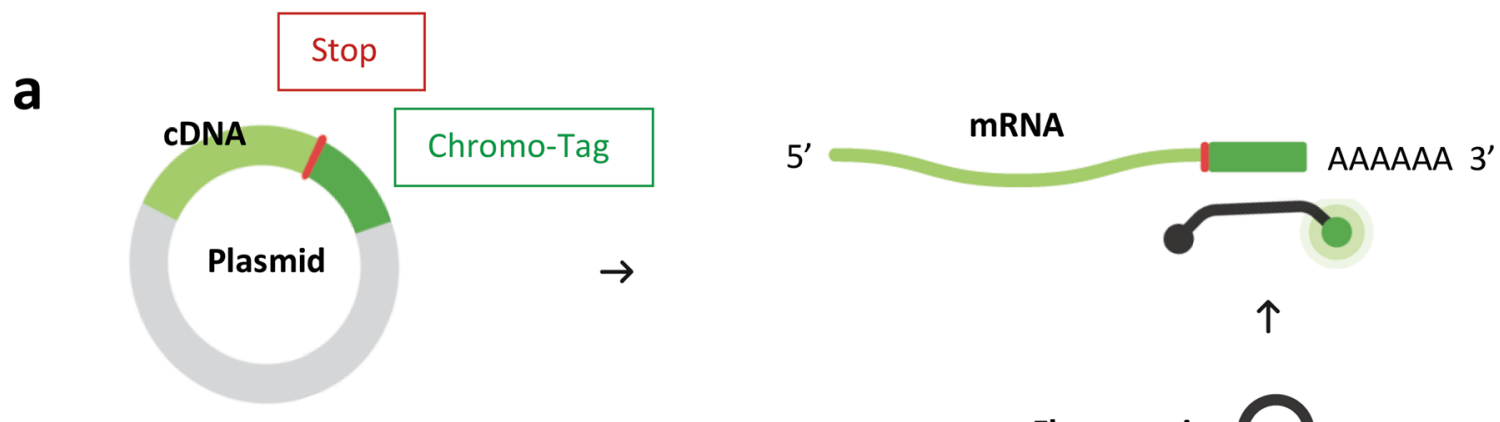

$\begin{aligned} & \text { Fluorogenic } \\ & \text { Probe }\end{aligned}=$

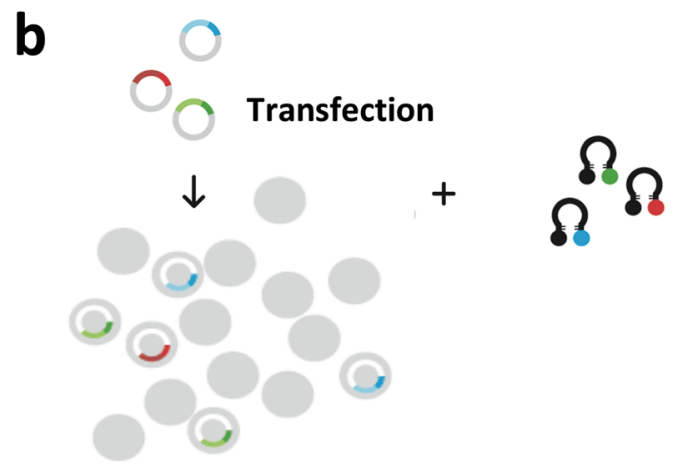

Fig. 4 Chromovert technology flowchart. a cDNAs are subcloned for expression of mRNAs comprising $3^{\prime}$ untranslated plasmid-encoded Chromo-Tag sequences for detection using fluorogenic oligonucleotide signaling probes. Protein expression products remain untagged. b To create cell lines, one or

and isolate those cells that are compatible with the viable and functional expression of biological targets including proteins that are otherwise cytotoxic in cell cultures.

\section{Materials and methods}

Production of cell lines

Each gene of interest was subcloned into an independent mammalian expression vector containing a Chromo-Tag and drug selectable marker and transfected into HEK 293T and CHO cells cultured using DMEM supplemented with $10 \%$ fetal bovine serum, a $4 \mathrm{mM}$ L-Glutamine, $10 \mathrm{mM}$ Hepes $\mathrm{pH}$ 7.4. After

\section{Clonal \\ Cell Lines}

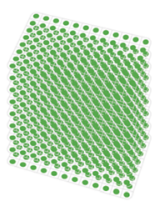

Flow

Cytometry

more Chromo-Tagged cDNAs are transfected into cells, the transfected cells are exposed to differentially-labeled signaling probes and individual positive cells are isolated using flow cytometry. Downstream testing is used to select final cell lines

1-3 days cells were transferred to fresh media containing mild selective pressure for 7-14 days. On the day of the experiment, each well of four to six 24-well tissue culture plates was plated with $200-400 \mu \mathrm{L}$ of transfected cells at $1.25-2 \times 10^{6}$ cells/mL in serum-free media and exposed to differentially-labeled signaling probes directed to the expression products. For each well, 1.7-2.5 $\mu \mathrm{L}$ of each probe at a concentration of $2.5-5 \mu \mathrm{M}$ was added to $50 \mu \mathrm{L}$ of serum free media in tube $\mathrm{A}$ and 0.3-0.75 $\mu \mathrm{L}$ of transfection reagent, using Lipofectamine for HEK cells (Thermo Fisher 18324) and jetPEI for CHO (Polyplus Transfection 101), added to tube $\mathrm{B}$. The contents of tubes $\mathrm{A}$ and $\mathrm{B}$ were mixed and incubated at room temperature for 25-30 min prior to addition to the well. Following a 1-2 $\mathrm{h}$ incubation at 
$37{ }^{\circ} \mathrm{C}$ in a tissue culture incubator, serum-free media was added to the well up to a total volume of $1 \mathrm{~mL}$. The plates were spun at $2000 \mathrm{RPM}$ for $4 \mathrm{~min} .750 \mu \mathrm{L}$ of media was aspirated and replaced with $250 \mu \mathrm{L}$ of fresh serum-free media. The cells were incubated for an additional $2-2.5 \mathrm{~h}$ and then used to isolate individual positive cells using Beckman Coulter Epic Altra or Becton Dickinson FACS Aria flow cytometers.

Fluorogenic probes directed to plasmid-encoded sequences downstream of the 3' stop codon of the cDNAs of interest were used. A first Chromo-Tag comprising portions of the reverse-complement of the mRNA sequence of the human $V a v$ gene and two derivate Chromo-Tag sequences were generated and used. Signaling probes directed to the Chromo-Tags are listed below including fluorophores and quenchers. BHQ2 was also used. 5-Methyl-dC and 2-Amino dA chemical modifications were included for the underlined bases except in probes used for the $\mathrm{ENaC}$ cell line.

Signaling probes directed to Chromo-Tags

Chromo-Tag A:5'-Cy5 GCCAGTCCCAGTTCCTG TGCCTTAAGAACCTCGC BHQ3-3’

\section{Chromo-Tag B:5'-CY5.5 GCGAGTCGCA GAACGACAGGGTTAACTTCCTCGC BHQ3-3' \\ Chromo-Tag C:5'-Fam GCGAGAGCGACAAG CAGACCCTATAGAACCTCGC BHQ1-3'}

Individual positive cells were expanded in 96-well multiter plates. Cell lines were binned based on growth rate, maintained using automated cell culture methods and functional cell-based assays were used to select final clones. The ENaC cell line was created without robotic cell culture methods and a custom low-sodium F-12 HAM media (Sigma N4888) formulation was used for cell culture (Shekdar and Langer 2017).

\section{Cell-based assays}

Functional testing using fluorescent cell-based assays of cells cultured in the absence of any antibioticmediated selectable pressure was performed over time (typically for at least 4-6 weeks) to identify inherently stable clones. Commonly available fluorescent membrane potential and calcium flux kits and reagents were used for fluorescent cell-based assays in 96-well and 384-well plates using the Molecular Devices FLIPR 3 and Hamamatsu FDSS 6000 plate readers as described (Shekdar and Langer 2011, 2017; Shekdar et al. 2012a, b). Electrophysiology of ion channel cell lines was obtained using the Nanion Technologies Patchliner patch clamp system. The patch clamp recordings were conducted according to Nanion's standard procedure (Obergrussberger et al. 2014). Currents were measured using the whole-cell configuration. The extracellular solution contained the following: $140 \mathrm{mM} \mathrm{NaCl}, 4 \mathrm{mM} \mathrm{KCl}, 1 \mathrm{mM} \mathrm{MgCl}, 2 \mathrm{mM}$ $\mathrm{CaCl}_{2}, 5 \mathrm{mM}$ glucose, $10 \mathrm{mM}$ Hepes, $\mathrm{pH} 7.4$ (adjusted with $\mathrm{NaOH}$ ). The intracellular solution for sodium current recordings contained the following: $50 \mathrm{mM} \mathrm{CsCl}, 10 \mathrm{mM} \mathrm{NaCl}, 60 \mathrm{mM} \mathrm{CsF}, 20 \mathrm{mM}$ EGTA, 10 mM Hepes, pH 7.2 (adjusted with $\mathrm{CsOH}$ ). For transient transfection studies, Fugene 6 (Promega E2691) was used to transfect expression plasmid into cells cultured as above and cells were assayed three days post-transfection.

Acknowledgements In loving memory of Chromocell cofounder and Rockefeller University Professor Günter Blobel, doctoral advisor to Kambiz Shekdar, and with deep gratitude to Chromocell co-founder and CEO Christian Köpfli, Research Foundation to Cure AIDS Chairwoman Karen Hagberg, Columbia University Medical Center/New York-Presbyterian Hospital Chairman of Medicine Don Landry, and all colleagues and advisors.

Author contributions KS conceptualized and performed the first proof-of-concept experiments for Chromovert technology and Chromo-Tags and conceptualized cell-based proteolysis of ENaC. DS optimized Chromovert technology. JL performed flow cytometry and experiments. JA, AL, LS, PS, OB, OD, and $\mathrm{SV}$ performed experiments. All authors contributed to data analysis. KS wrote the manuscript.

Funding This work was privately funded by Chromocell.

Data availability Data generated and analyzed in this study are included in this publication. Materials are available from the corresponding author.

\section{Compliance with ethical standards}

Conflict of interest KS is CSO, co-founder and director of Chromocell Corporation with equity in the company, sole member of Secondcell Bio, LLC established to make Chromovert Technology available to the research community and president of Research Foundation to Cure AIDS, with a license to the technology in the field of curing HIV infection. DS is founder of D3 Insight Consulting, LLC. All authors performed their work as employees of Chromocell. 
Ethical approval Human sensory studies were performed according to protocols approved by an independent institutional review board.

Informed consent Human sensory studies were performed with written informed consent obtained from all participants.

\section{References}

Babich O, Garyantes T, Luo R, Palling D, Venkatachalan S, Wang-Fischer Y (2016) Sodium channel modulators for the treatment of pain and diabetes. US Patent 9458118

Chalfie M, Tu Y, Euskirchen G, Ward WW, Prasher DC (1994) Green fluorescent protein as a marker for gene expression. Science 263:802-805

Chandrashekar J, Mueller KL, Hoon MA, Adler E, Feng L, Guo W, Zuker CS, Ryba NJ (2000) T2Rs function as bitter taste receptors. Cell 100:703-711

Cockett M, Bebbington CR, Yarranton GT (1990) High level expression of tissue inhibitor of metalloproteinases in Chinese hamster ovary cells using glutamine synthetase gene amplification. Biotechnology 8:662-667

Eisenhut M, Shin J (2020) Pathways in the pathophysiology of coronavirus 19 lung disease accessible to prevention and treatment. Front Physiol 11:872

Fornius M (2013) Treatment of pulmonary edema by ENaC activators/stimulators. Curr Mol Pharmacol 6:13-27

Johnston G (2005) GABA(A) receptor channel pharmacology. Curr Pharm Des 11:1867-1885

Krautwurst D, Yau KW, Reer RR (1998) Identification of ligands for olfactory receptors by functional expression of a receptor library. Cell 95:917-926

Lemna et al (1990) Mutation analysis for heterozygote detection and the prenatal diagnosis of cystic fibrosis. N Engl J Med 322:291-296

Marras SA, Kramer FR, Tyagi S (1999) Multiplex detection of single-nucleotide variations using molecular beacons. Genet Anal 14:151-156

Matalon S, Bartoszewski R, Collawn JF (2015) Role of epithelial sodium channels in the regulation of lung fluid homeostasis. Am J Physiol Lung Cell Mol Physiol 309:L1229-1238

Mohan C, Park SH, Chung JY, Lee GM (2007) Effect of doxycycline-regulated protein disulfide isomerase expression on the specific productivity of recombinant $\mathrm{CHO}$ cells: thrombopoietin and antibody. Biotechnol Bioeng 98:611-615

Nowogrodzki A (2019) The challenge of using CRISPR to knock in genes The Scientist. https://www.the-scientist. com/lab-tools/jacking-up-gene-knock-ins-65504
Obergrussberger A, Haarmann C, Rinke I, Becker N, Guinot D, Brueggemann A, Stoelzle-Feix S, George M, Fertig N (2014) Automated patch clamp analysis of nACh $\alpha 7$ and $\mathrm{Na}(\mathrm{V}) 1.7$ channels. Curr Protoc Pharmacol 65(1):1-48

Offermanns S, Simon M (1995) $\mathrm{G} \alpha_{15}$ and $\mathrm{G} \alpha_{16}$ couple a wide variety of receptors to phospholipase C. J Biol Chem 270:15175-15180

Olsen RW, Sieghart W (2008) International union of pharmacology LXX subtypes of $\gamma$ aminobutyric acid A receptors: classification on the basis of subunit composition, pharmacology, and function. Update Pharmacol Rev 60:243-260

O’Malley HA, Isom LL (2015) Sodium channel $\beta$ subunits: emerging targets in channelopathies. Annu Rev Physiol 77:481-504

Pear WS, Nolan GP, Scott ML, Baltimore D (1993) Production of high-titer helper-free retroviruses by transient transfection. PNAS 90:8392-8396

Roper SD (2015) The taste of table salt. Pflugers Arch 467:457-463

Shekdar K, Langer J (2011) Cell lines expressing GABA receptor and methods of using them US Pat Publ 2011/0003711

Shekdar K, Langer J (2017) Cell lines expressing ENaC and methods of using them. US Patent 9534035

Shekdar K, Sawchuk D, Shah P (2012) Novel cell lines and methods. US Patent Publ 2012/0015841

Shekdar K, Venkatachalan S, Langer J, Sawchuk D (2012) Cell lines expressing CFTR and methods of using them. US Patent Publ 2012/0058918

Urlaub G, Emmanuel K, Carothers AM, Chasin LA (1983) Deletion of the diploid dihydrofolate reductase locus from cultured mammalian cells. Cell 33:405-412

Wang D, Tai PWL, Gao G (2019) Adeno associated virus vector as a platform for gene therapy delivery. Nat Rev Drug Discov 18:358-378

Wetzel H, Oles M, Wellerdieck C, Kuczkowiak M, Gisselmann G, Hatt H (1999) Specificity and sensitivity of a human olfactory receptor functionally expressed in human embryonic kidney 293 cells and xenopus laevis oocytes. J Neurisci 19:7426-7433

Zuker M (2003) Mfold web server for nucleic acid folding and hybridization prediction. Nucleic Acids Res 31:3406-3415

Publisher's Note Springer Nature remains neutral with regard to jurisdictional claims in published maps and institutional affiliations. 